\title{
Natural Course of the American Thyroid Association Response to Therapy Statuses (Dynamic Risk Stratification) in Differentiated Thyroid Cancer
}

\author{
Noha Mukhtar Hadeel Aljamei Abeer Aljomaiah Yosra Moria \\ Ali S. Alzahrani \\ Department of Medicine, King Faisal Specialist Hospital \& Research Centre, Riyadh, Saudi Arabia
}

\section{Keywords \\ Thyroid cancer · Papillary thyroid cancer · Tumor node metastasis staging · American Thyroid Association staging · Response to therapy}

\begin{abstract}
The concept of response to therapy in differentiated thyroid cancer (DTC) was introduced as a dynamic risk stratification used to assess the status of the disease at the time of the evaluation during the follow-up and the risk of recurrence in the future. Our aim in this study was to evaluate the natural course over time of different response to therapy statuses.

Methods: We studied 501 nonselected DTC patients (102 males and 399 females) with a median age of 37 years (interquartile range [IQR] 29-48). All patients underwent near-total or total thyroidectomy followed by $1-131$ ablation (initial management). Results: Of the 501 patients, 387 patients (77.2\%) did not have any additional therapuetic interventions after the initial management. In this group, the response to therapy status at the time of the first evaluation after I-131 (median 17 months, IQR 14-22) was an excellent response in $258(66.7 \%)$, an indeterminate response in 101 (26.1\%), biochemically incomplete in $17(4.4 \%)$, and structurally incomplete in 11 patients (2.8\%). The status changed
\end{abstract}

spontaneously without any intervention in many of them. At the last follow-up visit (median duration 101 months, IQR 71-126), 357 patients (92.2\%) achieved an excellent response, $4(1 \%)$ an indeterminate response, $8(2.1 \%)$ a biochemically incomplete status, $16(4.1 \%)$ a structurally incomplete status, and $2(0.5 \%)$ died secondary to DTC with a structurally incomplete status. The response to therapy in the other 114 patients who underwent additional interventions changed from before intervention to the last evaluation as follows: excellent response, 0 to 60 patients (52.6\%), indeterminate response, $20(17.5 \%)$ to 1 patient $(0.9 \%)$, biochemically incomplete $25(21.9 \%)$ to 10 patients (9\%), and structurally incomplete $69(60.5 \%)$ to 43 patients (37.7\%). Overall, at the last evaluation, 417 (83.2\%) were in an excellent response, $5(1 \%)$ in an indeterminate response, $18(3.6 \%)$ in a biochemically incomplete status, $50(10.2 \%)$ in a structurally incomplete status, and 11 (2.2\%) died secondary to DTC with a structurally incomplete status. Conclusions: The response to therapy at the initial evaluation is predictive of the longterm outcome. Most patients with the indeterminate response and some in the biochemically incomplete statuses spontaneously regress to an excellent status. Mortality and progression of DTC occur mostly in the structurally incomplete status.

(c) 2020 European Thyroid Association Published by S. Karger AG, Basel

\footnotetext{
karger@karger.com

www.karger.com/et

(c) 2020 European Thyroid Association

Published by S. Karger AG, Basel
}

Karger"

Ali S. Alzahrani

MBC-46 (Department of Medicine)

PO Box 3354

Riyadh 11211 (Saudi Arabia)

aliz@kfshrc.edu.sa 


\section{Introduction}

Differentiated thyroid cancer (DTC) is the most common endocrine malignancy and its incidence has been increasing over the last 4 decades [1-4]. Although some data suggest a real increase in incidence [3], it is generally attributed to better detection methods $[2,4,5]$. The widespread use of neck ultrasonography and other imaging modalities has resulted in high detection rates of thyroid nodules; a small but significant proportion of them are malignant [3-5]. The vast majority of those tumors are papillary thyroid cancer, and more than $70 \%$ are small in size and carry minimal risk for mortality [1]. Realizing these facts, many experts in the field proposed a change in the diagnostic and therapeutic approaches to these low-risk DTC [6-9]. Over the last decade, this better understanding of the pattern and clinical course of DTC has resulted in a paradigm shift in its management with the adoption of risk-tailored strategies based on the estimation of the risk of recurrence and mortality [8]. Tumors that are low risk are usually treated more conservatively with lobectomy and do not routinely receive radioactive iodine (I-131) while high-risk tumors are treated more proactively with total or near-total thyroidectomy with or without lymph node dissection and I-131 adjuvant therapy $[6,8]$. The risk stratification concepts were further extended to include follow-up evaluations after the initial management based on the available information at the time of evaluation, so-called dynamic risk stratification $[6,10,11]$. Dynamic risk stratification indicates that risk changes due to the institution of therapeutic measures and the natural course of the disease [8-10]. For example, a high-risk tumor at the time of initial management may turn to be of low risk at the time of follow-up. The 2015 American Thyroid Association (ATA) guidelines embraced and emphasized these concepts, which have become part of the DTC standard of care [6].

While conservative approaches to the management of low-risk patients are gaining more acceptance, there are significant variations in practice between different institutions and different countries. Total or near-total thyroidectomy with or without lymph node dissection followed by I-131 remnant ablation/adjuvant therapy remains the most commonly practiced management strategy for intermediate and high-risk patients and even for a good proportion of low-risk patients $[12,13]$. Patients are usually evaluated at 6-12 months after I-131 ablation/therapy for the response to therapy of the disease. The ATA guidelines classify the response to therapy into four categories [6]. An excellent response indicates no clinical, biochemical, or radiological evidence of the disease with suppressed serum $\mathrm{Tg}$ level $<0.2 \mathrm{ng} / \mathrm{mL}$ or stimulated thyroglobulin $(\mathrm{Tg})$ level of $<1 \mathrm{ng} / \mathrm{mL}$ in the absence of anti-Tg antibodies (Abs) and negative imaging studies. An indeterminate response refers to a situation when residual or recurrent disease cannot be confidently excluded with mild elevation of $\mathrm{Tg}$ (suppressed $\mathrm{Tg} 0.2$ to $<1 \mathrm{ng} / \mathrm{mL}$ or stimulated Tg 1 to $<10 \mathrm{ng} / \mathrm{mL}$ ), positive anti-Tg Abs, and/or some nonspecific findings on imaging studies. A biochemically incomplete status refers to a significant elevation of serum Tg (suppressed $\geq 1 \mathrm{ng} / \mathrm{mL}$ or stimulated $\geq 10 \mathrm{ng} / \mathrm{mL}$ ) in the absence of radiological or cytological evidence of the disease. A structurally incomplete status refers to the presence of disease on radiological, cytological, and/or histopathological investigations, usually accompanied by a significant elevation of serum Tg or anti-Tg Abs. The natural history of these different statuses over time is important for predicting what will happen to the patients and for implementing timely and cost-effective monitoring and intervention strategies. In this study, our primary objective was to study the natural course of the response to therapy statuses after the initial management over time. Our hypothesis is that the response to therapy at the first evaluation after the initial management is highly predictive of the course of the disease over time and those patients in an excellent response are expected to do well over many years, need less intense follow-up and can be considered for discharge after a few years of follow-up. By contrast, we hypothesized that patients with an incomplete response to therapy are destined for recurrence, progression, and death and this group needs close follow-up and interventions when appropriate. For that reason, we analyzed the long-term course of patients in any of these statuses at the first evaluation after I-131 ablation/therapy over many years of follow-up. In addition, we assessed the performance of the ATA and the American Joint Committee on Cancer Tumor Node Metastasis (AJCC TNM) staging system in our patients.

\section{Patients and Methods}

This study was undertaken at the King Faisal Specialist Hospital \& Research Center (KFSHRC), Riyadh, Saudi Arabia. An institutional review board approval was obtained from the Office of Research Affairs of KFSHRC. KFSHRC is the premier tertiary care institution and the main cancer care center in Saudi Arabia. Each year, 200-300 patients with newly diagnosed thyroid cancer are referred to KFSHRC for management and long-term follow-up. The management of DTC has been following international standards and guidelines since 1998. Except for very low-risk patients 


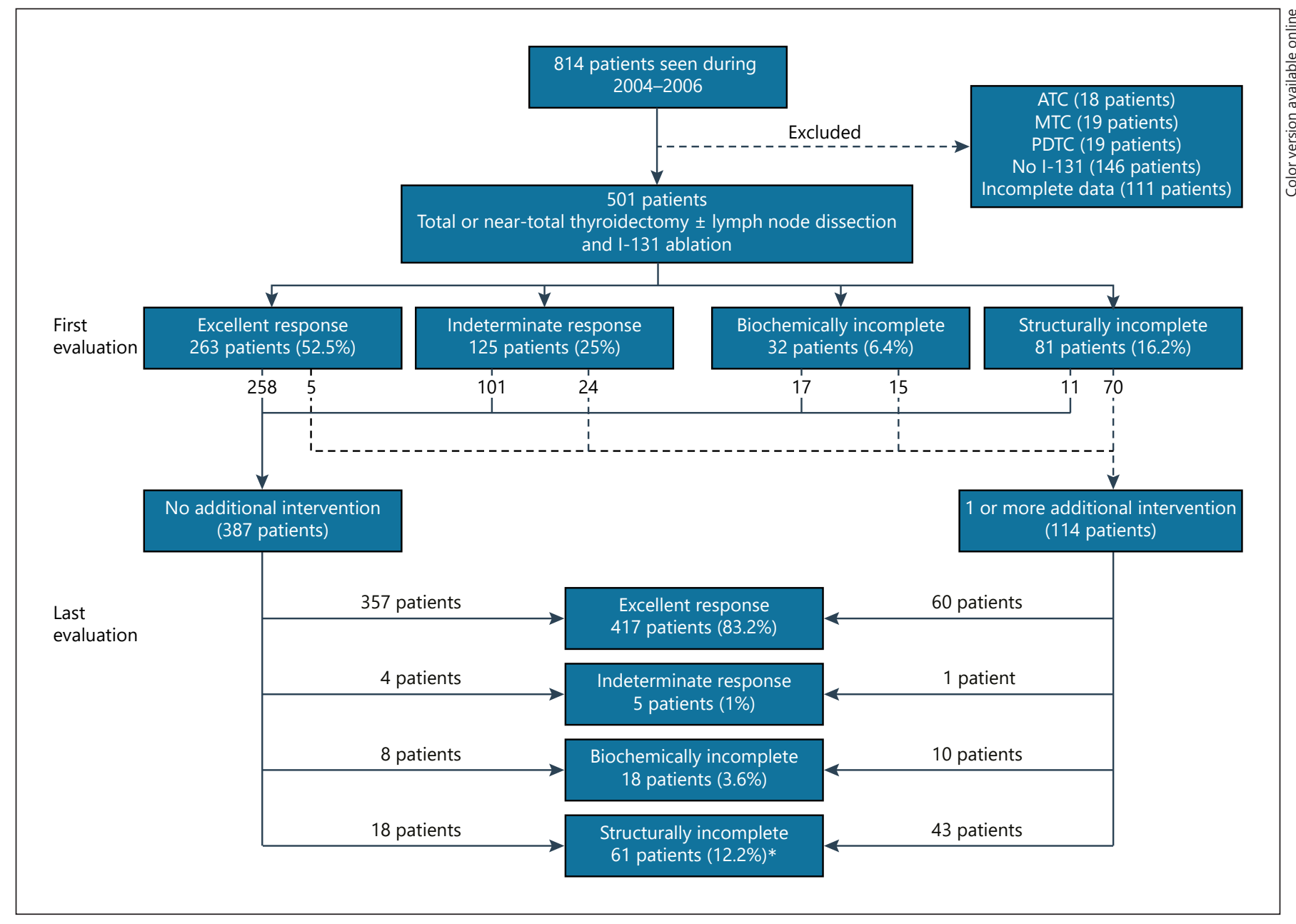

Fig. 1. Overall design and the results of the study showing the initial number of patients, the excluded patients, the initial management, response to therapy at the first evaluation after thyroid surgery and I-131 ablation, the additional interventions for a subgroup of 114 patients, and the final response to therapy statuses at the last follow-up.

(T1N0M0), our practice had been in the form of near-total or total thyroidectomy and therapeutic lymph node dissection if pathological lymph nodes are found on preoperative evaluation or intraoperative examination. Radioactive iodine (I-131) ablation had been given to most cases except for very low-risk patients (T1aN0M0). Thyroid hormone suppression was practiced aiming at serum TSH level of $0.1-0.5 \mathrm{mU} / \mathrm{L}$ for low to intermediate risk and $<0.1 \mathrm{mU} / \mathrm{L}$ for high-risk patients. Patients were usually evaluated 6-18 months after I-131 ablation with measurement of serum TSH, FT4, Tg, and anti-Tg Abs, and by neck ultrasonography. I-123 diagnostic whole-body scan and stimulated Tg levels were usually done once after I-131 ablation/therapy for patients with high and intermediate risk features without evidence of distant metastasis and more frequently as a preparation for more I-131 therapies for those with distant metastasis. Patients in an excellent response or an indeterminate response were usually seen on a yearly basis for follow-up, mostly with suppressed Tg, anti-Tg Abs, TSH, FT4, and neck ultrasonography. Other imaging studies (in- cluding computed tomography (CT) of the lungs and neck, combined CT/F-18 flurodeoxyglucose positron emission tomography and other imaging as appropriate) and more frequent monitoring were followed in patients with evidence of local disease or distant metastasis.

To ensure that we have an adequate long-term follow-up period for this study, we studied all patients diagnosed during the period January 2004-December 2006. A total of 814 patients with thyroid cancer were diagnosed at our institution during this period (Fig. 1). Considering the aim of the study of assessing the natural course of different ATA statuses and in order to ensure a homogeneous population that represents the majority of patients in the usual clinical practice, we excluded patients with anaplastic (18 patients), poorly differentiated (19 patients), and medullary thyroid cancers (19 patients). For the same reasons, we also excluded patients who did not receive I-131 (146 patients) since the definitions of different ATA response to therapy statuses depend to a great extent on serum $\mathrm{Tg}$ with different cutoff values for patients 
who received I-131 compared to those who did not. Finally, we excluded patients who did not have adequate follow-up data or were lost to follow-up (111 patients). A total of 501 cases of DTC remained and were analyzed for the objectives of this study (Fig. 1).

\section{Biochemical Measurements}

Serum Tg level, anti-Tg Abs, TSH, and FT4 were all performed by electrochemiluminescence assays on Cobas e 801 immunoassay analyzer (Roche Diagnostics $\mathrm{GmbH}$, Mannheim, Germany). The $\mathrm{Tg}$ assay has a lower limit of detection of $0.02 \mathrm{ng} / \mathrm{mL}$.

\section{Statistical Analysis}

The Statistical Package for the Social Sciences (SPSS) version 21 (IBM, Chicago) was used for the analysis. Numerical values are expressed as median and interquartile range (IQR) and categorical values as percentages and ratios. Kaplan-Meier analysis and logrank test were used to analyze the relationship between ATA staging and the AJCC TNM8 and the long-term outcome. They were also used to study the changes over time of the different response to therapy statuses. A $p$ value of $<0.05$ was considered significant.

\section{Results}

\section{Characteristics and Initial Management}

We studied 501 patients (102 males and 399 females) with a median age of 37 years (IQR 29-48). The clinical and pathological characteristics are summarized in Table 1 . The overall study design and the main results are summarized in Figure 1.

\section{ATA and AJCC TNM Staging Systems Predict the Response to Therapy at First Evaluation and Last Follow-Up}

The majority of patients were in low (246 patients) to intermediate (199 patients) ATA stages (Table 1). At the first evaluation after the initial management, 64.2 and $25.6 \%$ of patients in ATA low stage and 48.7 and $27.6 \%$ of the ATA intermediate stage achieved an excellent response and an indeterminate response, respectively ( $\mathrm{Ta}-$ ble 2). By contrast, the majority of patients in the ATA high-stage DTC had either structurally incomplete $(58.9 \%)$ or biochemically incomplete $(14.3 \%)$ statuses (Table 2).

Similarly, 95.1 and $83.4 \%$ of the patients in the ATA low and intermediate stages achieved an excellent response at the last follow-up evaluation. In comparison, only $30.4 \%$ of patients in the ATA high stage achieved an excellent response, and $66.1 \%$ continued to have a structurally incomplete response, including $17.9 \%$ of them who died secondary to structural disease (Table 2). The ATA staging was highly predictive of the long-term outcome (Fig. 2a).

Response to Therapy in DTC
Table 1. Clinical and pathological features of 501 patients with DTC

\begin{tabular}{lc}
\hline Characteristic & $N(\%)$ \\
\hline Median age (range), years & $37(6-76)$ \\
Sex (male:female) & $102: 399$ \\
Median tumor size (range), cm & $2.0(0.8-13)$ \\
Tumor type, $n$ (\%) & \\
$\quad$ Classic PTC & $361(72)$ \\
Follicular variant PTC & $82(16.3)$ \\
Tall cell variant PTC & $17(3.4)$ \\
Diffuse sclerosing variant PTC & $5(1.0)$ \\
Follicular thyroid cancer & $13(2.6)$ \\
Hurthle cell cancer & $9(1.8)$ \\
Other rare types & $14(2.8)$ \\
Tumor multifocality & $222(44.3 \%)$ \\
Extrathyroidal invasion & $272(54.3 \%)$ \\
Lymphovascular invasion & $100(20 \%)$ \\
Lymph node metastasis & $227(45.3 \%)$ \\
Distant metastasis & $48(9.6 \%)$ \\
ATA risk staging, $n$ (\%) & \\
Low risk & $246(49)$ \\
Intermediate & $199(39.7)$ \\
High & $56(11.2)$ \\
AJCC TNM8, $n$ (\%) & \\
Stage I & $416(83)$ \\
Stage II & $57(11.4)$ \\
Stage III & $4(0.8)$ \\
Stage IVb & $24(4.8)$ \\
Median I-131 administered activity (mCi) & $147(29-300)$ \\
Additional interventions & $114(22.8 \%)$ \\
\hline & \\
& \\
&
\end{tabular}

DTC, differentiated thyroid cancer; ATA, American Thyroid Association; AJCC TNM, American Joint Committee on Cancer Tumor Node Metastasis; PTC, papillary thyroid cancer

AJCC TNM staging also was predictive of the outcome at first follow-up and last evaluation after the initial management (Table 2). At the first evaluation, around $60 \%$ of stage I were in an excellent response while $70.8 \%$ of stage IV had a structurally incomplete disease. At the last evaluation, $93.3 \%$ and $49.1 \%$ of stage I and II were in an excellent response while $100 \%$ and $91 \%$ of stage III and IV had a structural disease (Table 2). AJCC TNM staging was also predictive of the long-term outcome although less distinctly than ATA stages as some overlap between AJCC TNM stages occurred (Fig. 2b).

\section{ATA Response to Therapy Status at the Time of the First Evaluation after the Initial Management}

At a median time of 17 months (IQR, 14-22) after I-131 ablation/therapy, the ATA response to therapy statuses were as follows: 263 (52.5\%) were in an excellent 
Table 2. Response to therapy status at the first evaluation after initial management (thyroidectomy and I-131 ablation) and at the last evaluation against the ATA and AJCC TNM8 staging systems

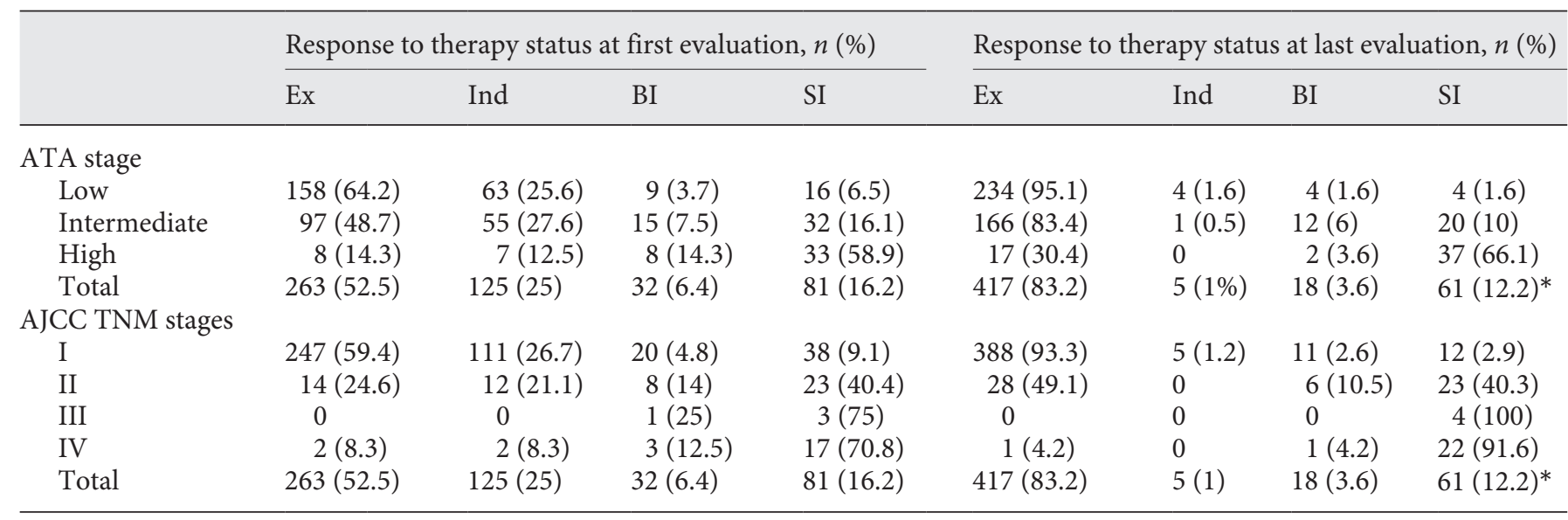

ATA, American Thyroid Association; AJCC TNM, American Joint Committee on Cancer Tumor Node Metastasis; Ex, excellent response; Ind, indeterminate response; BI, biochemically incomplete; SI, structurally incomplete. * Including 11 patients (2.2\%) who died from a structurally incomplete DTC.

response, $125(25 \%)$ in an indeterminate response, 32 $(6.4 \%)$ in a biochemically incomplete status, and 81 $(16.2 \%)$ in a structurally incomplete status (Fig. 1). Of the 501 patients, $114(22.8 \%)$ had additional interventions in the form of additional surgeries, I-131, external radiotherapy, and/or ethanol injections (online suppl. Table 1; see www.karger.com/doi/10.1159/000511708 for all online suppl. material). To avoid selection bias, we did not exclude these patients, but in order to eliminate the effect of the additional interventions on the status, we assessed the status of those patients before any additional interventions took place (online suppl. Table 3).

\section{Natural Course of Patients Who Had No Additional Interventions}

As can be seen in Figure 1, 387 (77\%) patients did not have any additional interventions after the initial management (total or near-total thyroidectomy \pm lymph node dissection and I-131 ablation/therapy). The ATA response to therapy statuses at the time of first evaluation after I-131 (median 17 months, IQR 14-22) was 258 $(66.7 \%)$ in an excellent response, $101(26.1 \%)$ in an indeterminate response, $17(4.4 \%)$ in a biochemically incomplete status, and $11(2.8 \%)$ in a structurally incomplete status (see online suppl. Table 2). The ATA statuses changed spontaneously without any intervention in many of them, especially those in the indeterminate group. At the last follow-up visit (median duration 101 months, IQR 71-126), 357 patients (92.2\%) were in an excellent response, $4(1 \%)$ in an indeterminate response, $8(2.1 \%)$ in a biochemically incomplete status, $16(4.1 \%)$ in a structurally incomplete status, and $2(0.5 \%)$ died secondary to thyroid cancer with a structurally incomplete status (online suppl. Table 2).

\section{Disease Course and Response to Therapy Status Changes in Patients Who Had Additional \\ Interventions}

After the initial management, 114 patients had additional interventions (online suppl. Table 1). At a median duration of 12 months (IQR 12-19) after the initial management, $5(4.4 \%)$ patients were in an excellent response, $24(21.1 \%)$ in an indeterminate response, $15(13.2 \%)$ in a biochemically incomplete response, and $70(61.4 \%)$ in a structurally incomplete response (online suppl. Table 3 ).

The response to therapy status before any additional intervention (median duration after I-131 ablation/therapy was 14 months, range 11-23) was as follows: none in an excellent response, 20 (17.5\%) in an indeterminate response, $25(21.9 \%)$ in a biochemically incomplete status, and $69(60.5 \%)$ in a structurally incomplete response (online suppl. Table 3). After 1 or more interventions (online suppl. Table 1), the response to therapy status at the last follow-up visit of those patients were as follows: $60 \mathrm{pa}$ tients (52.6\%) achieved an excellent response, 1 (0.9\%) an indeterminate response, 10 (8.8\%) biochemically incomplete, and $43(37.7 \%)$ structurally incomplete statuses (online suppl. Table 3 ). 


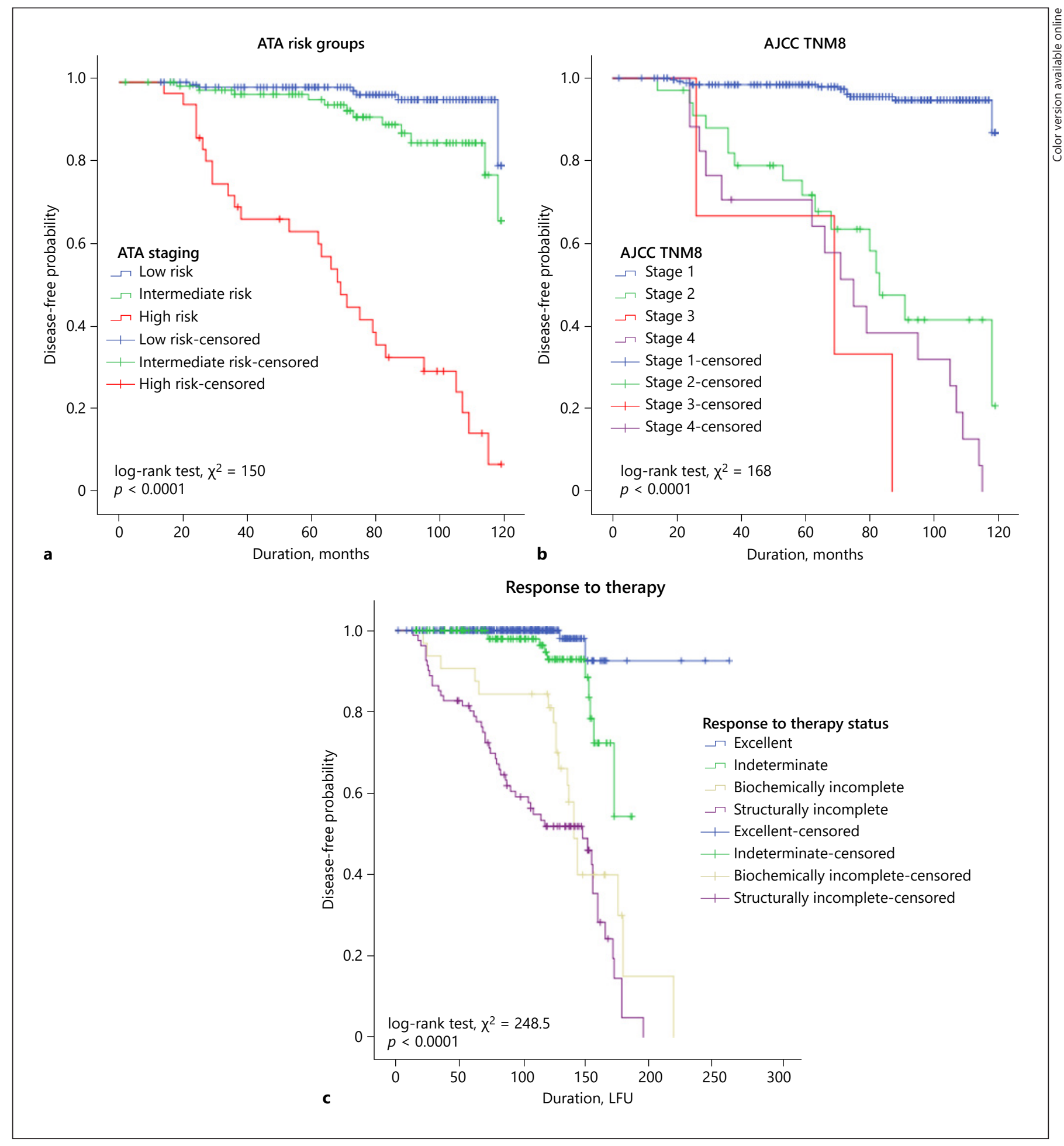

Fig. 2. Kaplan-Meier analyses showing the disease-free probabilities based on the ATA risk stratification (a), the AJCC/TNM8 stages (b) and response to therapy statuses at the first evaluation after initial management (c). All of these risk stratification and staging systems correlated well with the final outcome (median duration of 105 months [IQR 71-132] after I-131 ablation/therapy). ATA, American Thyroid Association; AJCC TNM, American Joint Committee on Cancer Tumor Node Metastasis. 
Fig. 3. A summary diagram showing the changes in the numbers and percentages of patients in different response to therapy statuses at the first evaluation after the initial management at a median time of 17 months (IQR 14-22) after I-131 ablation and at the last follow-up visit (median duration 105 months [IQR 71-132] after I-131 ablation). IQR, interquartile range. * Including 11 patients who died with a structurally incomplete status.

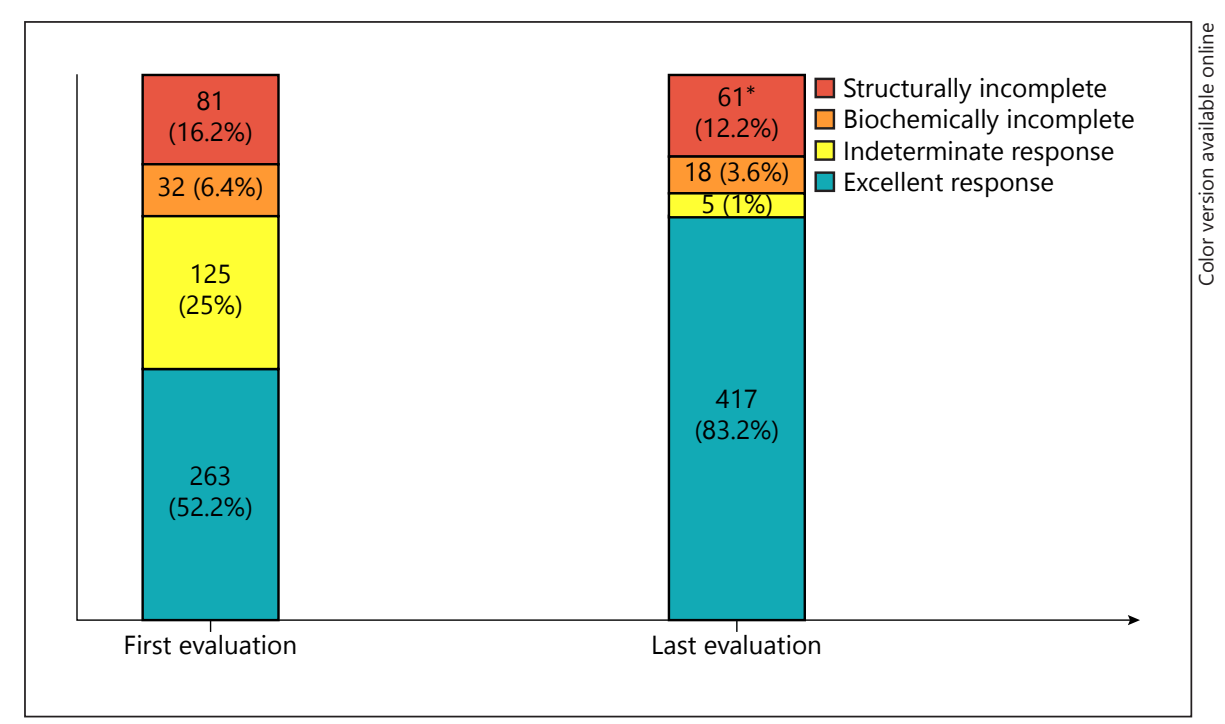

Overall Course of the Response to Therapy Statuses

At a median duration of 105 months (IQR 71-132) after I-131 ablation/therapy, the change of the response to therapy status from the time of the first evaluation after the initial management to the last visit of the 501 patients including those who had no additional intervention (387 patients) and those who had 1 or more additional interventions (114 patients) was as follows (Fig. 3): excellent status, 263 patients (52.5\%) at initial evaluation increased to $417(83.2 \%)$ at last visit; indeterminate status, 125 patients $(25 \%)$ decreased to 5 patients $(1 \%)$; biochemically incomplete, 32 patients $(6.4 \%)$ decreased to 18 patients (3.6\%); and structurally incomplete, 81 patients (16.2\%) decreased to 61 patients (12.2\%) including 11 patients $(2.2 \%)$ who died with structurally incomplete disease. The response to therapy status at the first evaluation after the initial management was predictive of the response to therapy statuses at the last follow-up (Fig. 2c). A major change occurred in the indeterminate status group. However, there was no significant difference in this change between those who were labeled to have an indeterminate status based on positive anti-Tg antibodies (36/38 patients achieved excellent response at the last follow-up) and those who had mildly elevated Tg or nonspecific changes on imaging studies (79/87 patients achieved an excellent response at the last follow-up) with $p$ value of 0.72 .

\section{Performance of Dynamic Risk Stratification in}

Patients Who Would Receive RAI in the Current Era

Since our patients were managed at a time when most patients would have had total thyroidectomy and received I-131 even if they had low-risk disease, we attempt- ed to analyze the data for patients that would receive these modalities of treatment in current practice. Therefore, in a sub-analysis to test the performance of dynamic risk stratification in the current era, we excluded patients with $\mathrm{T} 1$ and $\mathrm{T} 2$ disease (T1N0M0 and T2N0M0) and included patients with T3, T4 tumors and/or those with lymph node or distant metastasis regardless of the tumor stage. Currently, in most centers around the world and in accordance with the ATA guidelines [6], those patients would undergo total or near-thyroidectomy and would receive I-131 ablation. In this sub-analysis, 291 patients with T3-T4 disease and/or with lymph node or distant metastasis were included. At the initial evaluation 6-18 months after I-131 ablation, 121 (41.6\%) were in an excellent status, $72(24.7 \%)$ in an indeterminate status, 26 $(8.9 \%)$ in a biochemically incomplete status, and 72 $(24.7 \%)$ in a structurally incomplete status. A total of 101 (34.7\%) patients had 1 or more additional interventions (additional surgery, I-131, XRT, and/or ethanol injections). At the last follow-up (median 113 months, IQR 75-139 months), 217 (74.6\%) were in an excellent response, $2(0.7 \%)$ in an indeterminate response, 14 (4.8\%) in a biochemically incomplete, $47(16.2 \%)$ in a structurally incomplete statuses, and 11 (3.8\%) died from DTC.

\section{Discussion}

In this study, we have assessed the natural course of patients with different ATA response to therapy statuses after the initial management. We have demonstrated that (1) there is a good correlation between the ATA and 
AJCC TNM8 staging systems and the outcome at the first evaluation after initial management and at the final evaluation, (2) the ATA response to therapy status at the first evaluation is predictive of the long-term outcome, (3) the majority of patients with an indeterminate status and a significant proportion of those in a biochemically incomplete status achieved an excellent response status over time without further intervention, and (4) disease progression and mortality occurred predominantly in high ATA stage and the structurally incomplete response group at the first follow-up evaluation visit after initial management.

The ATA and AJCC TNM staging systems are excellent tools to assess the risk of recurrence and mortality and their predictive power has been demonstrated in many studies from around the world $[8,9,14-18]$. This is again demonstrated in this study from a Middle Eastern population (Table 2). However, these staging systems are static systems that utilize clinicopathological features and radiological information available at the time of initial management and do not consider the possible changes in the clinical course over time and the additional biochemical and radiological data that become available during follow-up. This is where the concept of dynamic risk stratification was introduced which is based on the fact that the disease status may change over time, and the risk is a dynamic process that should be assessed at each visit $[6,8,10,17]$. Initially, dynamic risk stratification was suggested to be used during the first 2 years after the initial management [10]. However, studies have shown that this system is valid and robust at all stages of surveillance as it utilizes the available clinical, biochemical, and radiological data at any time of assessment during the follow-up $[9,14,19]$. Our study shows that this system is highly reliable in predicting the long-term outcome. More importantly, it shows also that the dynamic response to therapy risk stratification during the first 1-2 years highly correlates with the dynamic risk stratification at the last followup evaluation (online suppl. Table 2, 3; Fig. 3). In fact, the overall outcome of patients at the last follow-up was even better than at the first 1-2 years as many patients achieve excellent response either spontaneously (the majority of patients) or after additional therapies (Fig. 3; online suppl. Table 2, 3).

An indeterminate status is a situation where the presence or absence of the disease cannot be confidently confirmed $[6,8]$. It is characterized by mildly detectable $\mathrm{Tg}$ $(<1 \mathrm{ng} / \mathrm{mL}$ on thyroxine or $1-<10 \mathrm{ng} / \mathrm{mL}$ off thyroxine), elevated anti-Tg Abs that make Tg level unreliable as a tumor marker, and/or nonspecific imaging findings [6].
The ATA guidelines recommend expectant management of patients with an indeterminate response to therapy [6]. In our study, at the first evaluation after the initial management, over a quarter $(26.1 \%)$ of the patients who did not receive any further intervention were in an indeterminate response (online suppl. Table 2). Without any further intervention, only $1 \%$ of them continued to have an indeterminate status. The vast majority of them spontaneously achieved an excellent response (online suppl. Table 2; Fig. 1). This supports following a more expectant approach with observation and follow-up of this group of patients. Although some studies suggested a difference in the outcome between those labeled to have an indeterminate status based on positive anti-Tg Abs and those due to mildly elevated $\mathrm{Tg}$ or nonspecific changes on imaging studies [20,21], we did not find a significant difference between these 2 subgroups of indeterminate response to therapy. This might be due to the small numbers of patients with indeterminate response to therapy at the last follow-up (Fig. 3).

Similarly, patients who achieved an excellent response status at the first evaluation after the initial management continued to enjoy excellent long-term outcome (Fig. 1; Fig. 3). This indicates that recurrence of the disease is rare and most cases that develop structural disease during the follow-up had never been free of the disease (persistent disease). This also suggests that this large group of patients who are in an excellent response can be infrequently monitored with serum $\mathrm{Tg}$ and anti-Tg $\mathrm{Ab}$ and can be considered for discharge after a few years of follow-up. Patients with a biochemically incomplete status are presumed to have small volume disease that escapes the limits of detection of the commonly used imaging techniques, or their disease is in unusual sites that were not scanned or seen. However, $23 \%$ and $11 \%$ of them spontaneously regressed to excellent and indeterminate responses, respectively, while $29 \%$ of them progressed to structural disease (Fig. 1). The rest continued to have biochemical evidence of the disease without clear structural lesions.

Our study showed clearly that the group of patients that need more proactive monitoring and interventions are those with a structural disease since a significant percentage of them developed more progressive disease, and the vast majority of patients who died were in this category. This was also shown in previous studies. Therefore, close monitoring and appropriate interventions should be implemented as necessary in this group of patients. This is also supported by the fact that our study also shows that additional therapeutic interventions, when indicated, are 
effective in a good percentage of patients, as demonstrated in the 114 patients who received additional interventions in this study (online suppl. Table 3 ). In this subgroup, the percentage of patients who achieved an excellent response increased from zero before additional interventions to 60 patients (52.6\%) while the rates of biochemically incomplete and structurally incomplete statuses decreased from $21.9 \%$ and $60.5 \%$ before interventions to $9 \%$ and $37.7 \%$ at the last follow-up (online suppl. Table 3 ).

Although dynamic risk stratification has become part of the routine care of patients with DTC, certain issues remained of concern including the impracticality and non-convenience of doing stimulated Tg and I-123 whole body scans. However, these are not needed in most patients as definitions of the different statuses in non-stimulated situations exist and correlate well with stimulated studies [6]. Definitions of the different statuses were initially established for patients who undergo total or neartotal thyroidectomy and I-131 ablation [6]. However, currently more patients in the low and intermediate risk groups undergo lobectomy for DTC and do not receive I-131 ablation. For that reason, studies also have been performed to validate dynamic risk stratifications in patients undergoing lobectomy alone or total thyroidectomy without I-131 ablation [22-24].

Our study has some weaknesses and strengths. The study is retrospective and included patients at a time when the general approach for management of DTC was more in the form of "one size fits all." However, the patients included were consecutive nonselected and were managed in the usual standard approach at the time of the study. All of them had near-total or total thyroidectomy \pm lymph node dissection and I-131 ablation, and this minimized variation due to different management approaches. A large proportion of them (387 patients, $77 \%$ ) did not receive any additional therapy after the initial management, and this enabled us to study the natural history of different ATA statuses over a long period of follow-up without intervention. It also confirms the usability and generalizability of the dynamic risk stratification across different ethnic populations. To further assess the robustness of the dynamic risk stratification, we analyzed a large subgroup of our patients (291 patients) who had an intermediate to high risk disease (T3-T4 disease and/ or those with lymph node or distant metastases) and would have been treated with total or near-total thyroidectomy and I-131 ablation even in this era of the "minimalistic approach" to the management of DTC [6]. The results showed the ability of dynamic risk stratification at the first evaluation to predict the long-term outcome. It also shows that this is the group of patients in whom the risk of structural disease and mortality is high despite instituting aggressive management measures. Although the follow-up period in our study is long [median 105 months (IQR 71-132)], it is less than expected since the study included patients diagnosed in 2004-2006. The reason for this apparently shorter than expected follow-up time is that we have discharged a significant number of patients who have been in excellent response for years to decongest our practice in accordance with the ATA guidelines 2015 [6].

In summary, this study from a previously non-included ethnic group (the Middle East), shows that the "static" ATA and AJCC TNM staging systems perform very well in predicting the short- and long-term course of DTC and correlate well with the ATA response to therapy dynamic risk stratification system. The ATA response to therapy statuses in the first 1-2 years after the initial management predicts the long-term outcome and correlates well with ATA response to therapy statuses at the last evaluation. Most patients with an indeterminate response and a good percentage of those in the biochemically incomplete statuses spontaneously achieve excellent status over time without intervention, and an expectant management for these groups, especially the indeterminate status, is warranted. On the other hand, patients with a structurally incomplete status are at the highest risk of progressive disease and mortality and should be carefully monitored and managed.

\section{Acknowledgements}

The authors would like to thank colleagues at the Endocrine Section and Department of Medicine of the KFSHRC for their support.

\section{Statement of Ethics}

This study was undertaken at the King Faisal Specialist Hospital \& Research Center (KFSHRC), Riyadh, Saudi Arabia. An institutional review board approval was obtained from the Office of Research Affairs of KFSHRC (RAC\# 2130-015). Since the study was retrospective and involved only confidential medical record review without direct interaction with patients, a waiver of consent was granted by the Ethics Committee. All research included in this manuscript followed the guidelines for human studies and should include evidence that the research was conducted ethically in accordance with the World Medical Association Declaration of Helsinki. 


\section{Conflict of Interest Statement}

The authors have no conflicts of interest to declare.

\section{Funding Sources}

No specific funding was obtained for this study.

\section{Author Contributions}

Noha Mukhtar: conception of the research idea, data collection, tabulation, and analysis. Hadeel Aljamei: data collection, tabulation, and analysis. Abeer Aljomaiah: data collection, editing, and analysis. Yosra Moria: conception of the idea, analysis of the data, and review of the manuscript. Ali Alzahrani: conception of the research idea, supervising the whole research process, data analysis, and writing the manuscript.

\section{References}

1 Howlader N, Noone A, Krapcho M, Noone A, Neyman N, Aminou R, et al. SEER cancer statistics review, 1975-2009; 2012.

2 Kitahara CM, Sosa JA. The changing incidence of thyroid cancer. Nat Rev Endocrinol. 2016 Nov;12(11):646-53.

3 Kim J, Gosnell JE, Roman SA. Geographic influences in the global rise of thyroid cancer. Nat Rev Endocrinol. 2020 Jan;16(1):17-29.

4 Vigneri R, Malandrino P, Russo M. Is thyroid cancer increasing in incidence and aggressiveness? J Clin Endocrinol Metab. 2020 105(7):dgaa223

5 La Vecchia C, Negri E. Thyroid cancer: the thyroid cancer epidemic: overdiagnosis or a real increase? Nat Rev Endocrinol. 2017; 13(6):318

6 Haugen BR, Alexander EK, Bible KC, Doherty GM, Mandel SJ, Nikiforov YE, et al. American Thyroid Association Management Guidelines for Adult Patients with Thyroid Nodules and Differentiated Thyroid Cancer: the American Thyroid Association Guidelines Task Force on Thyroid Nodules and Differentiated Thyroid Cancer. Thyroid. 2016 Jan;26(1):1-133.

7 Luster M, Aktolun C, Amendoeira I, Barczyński M, Bible KC, Duntas LH, et al. European Perspective on 2015 American Thyroid Association Management Guidelines for adult patients with thyroid nodules and differentiated thyroid cancer: proceedings of an interactive international symposium. Thyroid. 2019 Jan;29(1):7-26.

8 Tuttle RM, Alzahrani AS. Risk stratification in differentiated thyroid cancer: from detection to final follow-up. J Clin Endocrinol Metab. 2019 Mar 15;104(9):4087-100.

9 Vaisman F, Tuttle RM. Clinical assessment and risk stratification in differentiated thyroid cancer. Endocrinol Metab Clin North Am. 2019 Mar;48(1):99-108.

10 Tuttle RM, Tala H, Shah J, Leboeuf R, Ghossein R, Gonen M, et al. Estimating risk of re- currence in differentiated thyroid cancer after total thyroidectomy and radioactive iodine remnant ablation: using response to therapy variables to modify the initial risk estimates predicted by the new American Thyroid Association staging system. Thyroid. 2010 Dec; 20(12):1341-9.

11 Momesso DP, Vaisman F, Yang SP, Bulzico DA, Corbo R, Vaisman M, et al. Dynamic risk stratification in patients with differentiated thyroid cancer treated without radioactive iodine. J Clin Endocrinol Metab. 2016;101(7): 2692-700.

12 Lamartina L, Durante C, Lucisano G, Grani G, Bellantone R, Lombardi CP, et al. Are evidence-based guidelines reflected in clinical practice? An analysis of prospectively collected data of the Italian thyroid cancer observatory. Thyroid. 2017 Dec;27(12):1490-7.

13 Owens PW, McVeigh TP, Fahey EJ, Bell M, Quill DS, Kerin MJ, et al. Differentiated thyroid cancer: how do current practice guidelines affect management? Eur Thyroid J. 2018 Nov;7(6):319-26.

14 Pitoia F, Jerkovich F, Urciuoli C, Schmidt A, Abelleira E, Bueno F, et al. Implementing the modified 2009 American Thyroid Association risk stratification system in thyroid cancer patients with low and intermediate risk of recurrence. Thyroid. 2015;25(11):1235-42.

$15 \mathrm{Kim}$ TH, Kim YN, Kim HI, Park SY, Choe JH, Kim JH, et al. Prognostic value of the eighth edition AJCC TNM classification for differentiated thyroid carcinoma. Oral Oncol. 2017;71:81-6.

16 Tuttle RM, Haugen B, Perrier ND. Updated American joint committee on cancer/tumornode-metastasis staging system for differentiated and anaplastic thyroid cancer (Eighth Edition): what changed and why? Thyroid. 2017 Jun;27(6):751-6.

17 Pitoia F, Jerkovich F. Dynamic risk assessment in patients with differentiated thyroid cancer. Endocr Relat Cancer. 2019;26(10): R553.

18 Shaha AR, Migliacci JC, Nixon IJ, Wang LY, Wong RJ, Morris LGT, et al. Stage migration with the new American Joint Committee on Cancer (AJCC) staging system (8th edition) for differentiated thyroid cancer. Surgery. 2019;165(1):6-11.

19 Hong CM, Lee WK, Jeong SY, Lee SW, Ahn BC, Lee J. Superiority of delayed risk stratification in differentiated thyroid cancer after total thyroidectomy and radioactive iodine ablation. Nucl Med Commun. 2014;35(11):1119-26.

20 Côrtes MCS, Rosario PW, Oliveira LFF, Calsolari MR. Clinical impact of detectable antithyroglobulin antibodies below the reference limit (Borderline) in patients with papillary thyroid carcinoma with undetectable serum thyroglobulin and normal neck ultrasonography after ablation: a prospective study. Thyroid. $2018 \mathrm{Feb} ; 28(2): 229-35$.

21 Oh H-S, Ahn JH, Song E, Han JM, Kim WG, Kim TY, et al. Individualized follow-up strategy for patients with an indeterminate response to initial therapy for papillary thyroid carcinoma. Thyroid. 2019 Feb;29(2):209-15.

22 Momesso DP, Vaisman F, Yang SP, Bulzico DA, Corbo R, Vaisman M, et al. Dynamic risk stratification in patients with differentiated thyroid cancer treated without radioactive iodine. J Clin Endocrinol Metab. 2016 Jul; 101(7):2692-700

23 Park S, Kim WG, Song E, Oh HS, Kim M, Kwon $\mathrm{H}$, et al. Dynamic risk stratification for predicting recurrence in patients with differentiated thyroid cancer treated without radioactive iodine remnant ablation therapy. Thyroid. 2017 Apr;27(4):524-30.

24 Park S, Jeon MJ, Oh H-S, Lee Y-M, Sung T-Y, Han $M$, et al. Changes in serum thyroglobulin levels after lobectomy in patients with lowrisk papillary thyroid cancer. Thyroid. 2018 Aug;28(8):997-1003. 